\title{
Aplicación del bioensayo EROD-H4IIE para la determinación de dioxinas en carnes de pollos broiler: un estudio de equivalencia con la cromatografía de gases de alta resolución acoplada a espectrometría de masas de alta resolución ${ }^{\#}$
}

\author{
Application of the EROD-H4IIE bioassay for the determination of dioxins \\ in broiler chicken meat: an equivalence study with high resolution gas chromatography \\ coupled to high resolution mass spectrometry \\ JT Schoffer ${ }^{\mathrm{a}}$, C Bustos-López ${ }^{\mathrm{b}}$, P Sotomayor ${ }^{\mathrm{a}}$, CA Mattar ${ }^{\mathrm{a}}$, A González ${ }^{\mathrm{a}}$, C Robles ${ }^{\mathrm{a}}$,

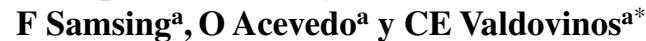 \\ ${ }^{a}$ Centro de Investigaciones Ecotoxicológicas, Facultad de Ciencias Silvoagropecuarias, Universidad Mayor, Santiago, Chile. \\ bepartamento de Ciencias Básicas, Facultad de Ciencias, Universidad Santo Tomás, Santiago, Chile.
}

\begin{abstract}
SUMMARY
The EROD bioassay with H4IIE cell line was applied in this study to determine the equivalence of the results for chicken meat between the ERODH4IIE Bioassay in pg TCDD-EQ/g of tissue, and the results of the gas chromatography coupled to high resolution spectrometry (HRGC/HRMS) in pg WHO-TEQ/g of fat of TEQs. 41 compound samples of chicken drumsticks were used. The samples were obtained in slaughtering plants of 4 different production facilities of Chile, during the sacrifice of animals (38 and 43 days old) between 2004 and 2007. Each sample was analysed with both analytical techniques. A regression model for the equivalence of both techniques was determined from the results. The model obtained was: HRGC/ HRMS $=0.481+0.051$ [EROD-H4IIE] $]^{2}, \mathrm{R}^{2}=0.885$, therefore for a value in EROD-H4IIE of $2.2 \mathrm{pg}$ TCDD-EQ/g of tissue it is estimated that it will correspond to $0.73 \mathrm{pg}$ WHO-TEQ/g of fat in HRGC/HRMS. Also, for the same value of EROD-H4IIE with a 95\% confidence, an estimate as an upper limit equal to $0.83 \mathrm{pg}$ WHO-TEQ/g of fat in HRGC/HRMS will be obtained. An upper limit equal to $0.88 \mathrm{pg} \mathrm{WHO-TEQ/g} \mathrm{of} \mathrm{fat} \mathrm{in} \mathrm{HRGC/HRMS} \mathrm{is}$ estimated by taking the same value of EROD-H4IIE with 99\% confidence. It is concluded that de EROD-H4IIE bioassay can be applied as a screening method in animal production systems and specifically in broiler chicken production.
\end{abstract}

Palabras clave: dioxina, bioensayo H4IIE, HRGC/HRMS, EROD.

Key words: dioxin, H4IIE bioassay, HRGC/HRMS, EROD.

\section{INTRODUCCIÓN}

Con el nombre genérico de dioxinas, se conoce a un grupo de compuestos orgánicos tricíclicos halogenados. El grado de cloración y la posición de los átomos de cloro determinan la existencia de 75 congéneres de dibenzodioxinas (PCDDs) y 135 de dibenzofuranos (PCDFs). Sólo los compuestos que son clorados en las posiciones 2, 3, 7 y 8 presentan toxicidad. Esto incluye 7 dibenzodioxinas, 10 dibenzofuranos y 12 bifenilos policlorinados (PCBs) (Roeder y col 1998). El compuesto de dioxina más tóxico es la 2,3,7,8-tetraclorodibenzo- $p$-dioxina (TCDD), también conocida con el nombre de dioxina Seveso (Whyte y col 2004).

En humanos y otros vertebrados, las dioxinas son consideradas como factores de riesgo para el desarrollo del cáncer (Fingerhut y col 1991), inmunodeficiencia (Weisglas-Kuperus y col 2000), menor tamaño de la próstata,

Aceptado: 12.05.2011.

\# Proyecto FONDEF D03I1035.

* Casilla 235 correo 34, Santiago, Chile; carlos.valdovinos@ umayor.cl reducción del peso testicular y de la espermatogénesis (EC 2001), disminución de los niveles séricos de testosterona (Egeland y col 1994), desarrollo de patologías del sistema nervioso central y periférico (Guo y col 2003), disrupción endocrina y mayor incidencia de diabetes (Longnecker y Michalek 2000, Sweeney y Mocarelli 2000), trastornos a la tiroides, alteraciones broncopulmonares (Nakanishi y col 1985) y otros.

Los seres humanos están expuestos a las dioxinas principalmente a través de la dieta: carne, productos lácteos, huevos y pescado (Spiro y Stigliani 2004, Saegerman y col 2006). Por tanto, debido a los casos de contaminación de carnes de aves de corral en Estados Unidos, la "Crisis de PCB/dioxinas de Bélgica" de 1999 (Hayward y col 1999, Covaci y col 2008), el hallazgo de dioxinas en carne de cerdo de Chile en 2008 (Valdovinos 2009) y el caso reciente en Alemania en 2010, que afectó a granjas avícolas y porcinas (PoultryMed), ${ }^{1}$ es necesario contar con una metodología de cribado (Unión Europea 2006)

http://www.poultrymed.com/Poultry/Templates/showpage.asp?D $\mathrm{BID}=1 \& \mathrm{LNGID}=1 \& \mathrm{TMID}=178 \& \mathrm{FID}=871 \& \mathrm{PID}=0 \& \mathrm{IID}=11684$. Fecha de consulta: 07 de enero de 2011. 
para la detección rápida y de bajo costo, previo al análisis químico confirmatorio.

Una manera efectiva de detección es a través de los efectos toxicológicos de estos contaminantes, que ocurren mediante el receptor de hidrocarburos aromáticos (AhR), factor de transcripción nuclear activado por unión de ligandos y que media la toxicidad del TCDD. El AhR en ausencia del ligando es citosólico, pero es transportado al núcleo cuando se activa con un ligando exógeno. La activación de AhR produce una serie de cambios bioquímicos en la célula, incluyendo la inducción del citocromo P4501A (CYP1A), utilizado para medir la actividad de la 7-etoxyresorufina- $O$-deetilasa (EROD) como biomarcador de exposición a dioxinas y compuestos similares a dioxinas (Richter y col 2001, Okey y col 2005), a través del bioensayo in vitro con la línea celular H4IIE. Por tanto, el objetivo del presente estudio es determinar la ecuación de equivalencia para los resultados en carnes de pollo, entre el bioensayo EROD-H4IIE en pg TCDD$\mathrm{EQ}^{2} / \mathrm{g}$ de tejido muestreado contra los resultados de la cromatografía de gases acoplada a espectrometría de masas de alta resolución (HRGC/HRMS) en pg WHO$\mathrm{TEQ} / \mathrm{g}$ de grasa, considerando a este último como método de referencia, mediante la determinación de un modelo de regresión, que permita estimar el valor esperado de contaminación por dioxinas que se obtendría a través de HRGC/HRMS si sólo se cuenta con los resultados del bioensayo EROD-H4IIE.

\section{MATERIAL Y MÉTODOS}

\section{MUESTRAS}

Para el presente estudio se utilizaron 41 muestras compuestas de trutros de pollo obtenidas en la planta faenadora de 4 planteles de Chile, codificados como P1, P2, P3 y P4, durante el beneficio de animales (38 y 43 días de edad) entre los años 2004 y 2007. Cada muestra compuesta se obtuvo en duplicado, a partir de muestras elementales constituidas por los trutros sin cuero y sin hueso de 10 animales del mismo origen. Las muestras fueron trituradas en la planta faenadora con una moledora industrial (BESTE ${ }^{\circledR}$ TK-12), obteniéndose 2 muestras de $250 \mathrm{~g}$ para el bioensayo EROD-H4IIE y 2 muestras de 250 g para el análisis HRGC/HRMS (muestra y contramuestra). Éstas fueron depositadas en bandejas de aluminio (previamente tratadas con acetona técnica al 99,5\%) y selladas, siendo congeladas a $-70{ }^{\circ} \mathrm{C}$ en el laboratorio a la espera de su análisis.
2 En este documento se utilizará TCDD-EQs para denominar los tóxicos equivalentes (TEQs) derivados del bioensayo EROD-H4IIE.
EXTRACCIÓN Y PURIFICACIÓN PARA APLICACIÓN DEL BIOENSAYO CELULAR H4IIE

De acuerdo al método descrito por Nicks y Tillitt (2003), se pesaron $20 \mathrm{~g}$ de muestra de carne de ave, adicionando una cantidad 3 veces superior al peso de la muestra $(60 \mathrm{~g})$ de sulfato de sodio $\left(\mathrm{Na}_{2} \mathrm{SO}_{4}\right)$ para el secado de líquidos existentes en la matriz. Posteriormente, se procedió a homogeneizar la muestra seca en una licuadora (Osterizer® 4172). El proceso de extracción de la grasa se realizó en un Soxhlet automático (VELP® Scientifica SER 148) durante 2 horas y 30 minutos, con una mezcla de diclorometano-hexano 1:1. La determinación del contenido lipídico del extracto se efectuó mediante cálculos gravimétricos a partir de la diferencia de peso de $1 \mathrm{~mL}$ del extracto, antes y después de ser sometido a calor para la evaporación de solventes y agua. El proceso de limpieza del extracto se realizó con el paso de la muestra a través de dos columnas de vidrio; la primera rellena con $\mathrm{Na}_{2} \mathrm{SO}_{4}$, silicato de potasio (KS), sílica 60:40 (sílica gel Grade 62, 60-200 mesh, $150 \AA$ A; SIGMA-ALDRICH ${ }^{\circledR}$ mezclada con $\mathrm{H}_{2} \mathrm{SO}_{4}$ ) y sílica 70:30 (sílica gel Davisil@ Grade 635, 60-100 mesh, 60 A; SIGMA-ALDRICH ${ }^{\circledR}$ mezclada con $\mathrm{H}_{2} \mathrm{SO}_{4}$ ) con diclorometano. La segunda columna de limpieza contenía $\mathrm{Na}_{2} \mathrm{SO}_{4}$, sílica gel 60 (sílica gel Grade 60, 70-230 mesh, 0.063-0.200 mm; Merck $\left.{ }^{\circledR}\right)$, KS y sílica 60:40 con una mezcla de diclorometano: hexano/3:97. La muestra obtenida fue reducida mediante el uso de rotaevaporador (Heidolph ${ }^{\circledR}$ Laborota 4000) alcanzando un volumen de $1 \mathrm{~mL}$ aproximadamente. Este volumen de muestra fue sometido al proceso de cambio de solvente, a través del uso de nitrógeno gaseoso, obteniéndose un extracto final de $150 \mu \mathrm{L}$ disuelto en isooctano. La totalidad de los solventes utilizados fue grado HPLC.

\section{BIOENSAYO CELULAR EROD-H4IIE}

Se aplicó el bioensayo EROD-H4IIE para la detección de PCDDs, PCDFs y DL-PCBs, según el protocolo descrito por Tillitt y col (1991) y modificado por Nicks y Tillitt (2003). Se utilizó la línea celular de hepatoma de Rattus norvegicus, importada de la American Type Culture Collection (ATCC ${ }^{\circledR}$, código CRL-1548 ${ }^{\mathrm{TM}}$ ). La línea celular fue cultivada en un medio D-MEM, enriquecido con un $15 \%$ de SFB, mantenida bajo condiciones estándares $\left(37^{\circ} \mathrm{C}\right.$, $5 \% \mathrm{CO}_{2}$ ) y se le permitió crecer de 4 a 5 días. Las células fueron sembradas en placas de microtítulo de 96 pocillos de fondo plano, Nunc ${ }^{\circledR}$, en un volumen de $300 \mu \mathrm{L} /$ pocillo con una densidad de $1,2 \times 10^{4}$ células/pocillo. Después de 24 horas de crecimiento, se procedió a dosificar el estándar de TCDD en 7 diluciones seriadas en razón 1:2 $(50 \mathrm{pg} /$ pocillo a $0,069 \mathrm{pg} /$ pocillo) en cuadruplicado. Dicho estándar fue usado para generar una curva de dosis respuesta con la cual todas las muestras fueron relacionadas.

Los extractos de las muestras se dosificaron con 7 diluciones en razón 1:3, en cuadruplicado. Junto con 
dichas muestras también se dosificaron, de igual manera, un control positivo (PC) correspondiente a tejido de Cyprinus carpio Linnaeus y un control negativo o matriz blanco (MB) correspondiente al Lepomis macrochirus Rafinesque, materiales de referencia del Columbia Environmental Research Center de Missouri, Estados Unidos. Además de estos controles se utilizó un blanco de procedimiento $(\mathrm{PB})$ correspondiente a una solución de diclorometano-hexano 1:1 (grado HPLC). Luego de la dosificación se dejó a las células bajo condiciones estándares por 72 horas para su posterior lectura. Para ello el medio fue removido a través de un lavador de placas (Thermo ${ }^{\circledR}$ Weelwash $4 \mathrm{Mk}$ 2), que utiliza agua ultrapura, dejando aproximadamente $60 \mu \mathrm{L}$ de medio/ pocillo. Terminado el lavado se incubó la placa por 5 minutos, para generar citólisis osmótica, dando como resultado la exposición del CYP1A1. Pasado el tiempo se retiró la placa de la incubadora y se le agregaron $20 \mu \mathrm{L}$ de buffer a $37^{\circ} \mathrm{C}$ con $80 \mu \mathrm{L}$ de dicumarol. Posteriormente, se adicionaron $20 \mu \mathrm{L}$ de $5 \mu \mathrm{M}$ de etoxiresorufina y $20 \mu \mathrm{L}$ de $5 \mu \mathrm{M}$ de NADPH. Finalmente, se colocaron las placas en un lector de fluorescencia (BioTek ${ }^{\circledR}$ FLx $800^{\mathrm{TM}}$ ), que realiza lecturas por 20 minutos con un filtro de excitación $530 \mathrm{~nm}$ y con un filtro de emisión de $580 \mathrm{~nm}$ para la lectura de la actividad EROD. Para la determinación de la proteína celular se realizó una lectura de 1 minuto con un filtro de excitación de $400 \mathrm{~nm}$ y un filtro de emisión de $460 \mathrm{~nm}$. Este método permite evaluar la actividad EROD y la proteína en el mismo pocillo (Kennedy y Jones 1994). En la etapa final del ensayo se utilizaron curvas de dosis respuestas para determinar la potencia relativa del extracto (RPFs), comparando los valores de las pendientes de los extractos de las muestras con los valores de la pendiente del estándar de TCDD para obtener los equivalentes tóxicos (TCDD-EQs) expresados en pg/g de muestra (Mason y col 1985, Tillitt y col 1993, Whyte y col 2004).

\section{CROMATOGRAFÍA DE GASES DE ALTA RESOLUCIÓN} ACOPLADA A ESPECTROMETRÍA DE MASAS DE ALTA RESOLUCIÓN

El análisis HRGC/HRMS fue realizado en el Research and Productivity Council de New Brunswick, Canadá. Para esto se utilizaron los métodos: US EPA Method 1613B, "Tetra-through Octa-Chlorinated Dioxins and Furans by Isotope Dilution HRGC/HRMS" y US EPA Method 8290A, "Polychlorinated Dibenzodioxins (PCDDs) and Polychlorinated Dibenzofurans (PCDFs) by High-Resolution Gas Chromatography/High-Resolution Mass Spectrometry (HRGC/HRMS)", cada uno con modificaciones leves (procedimientos estándares de RPC DX08 y DX09).

Se enriqueció la muestra ( 20 a 60 g) con una solución que contenía cantidades especificadas de cada uno de los 15 estándares internos de PCDD/PCDFs marcados isotópicamente (Wellington Laboratories Inc., Guelph,
Ontario, Canadá). Posteriormente, se realizó la extracción mediante Soxhlet por $16 \mathrm{~h}$ con diclorometano/hexano 1:1 (grado GC); luego se concentró en rotaevaporador (BÜCHI ${ }^{\circledR}$ RE 121 o Yamato ${ }^{\circledR}$ RE47). El contenido de grasa se determinó por análisis gravimétrico; el extracto se disolvió en hexano (grado GC). Luego se realizó una digestión usando sílica 70:30 (sílica gel 60, 100-200 particle size; Caledon ${ }^{\circledR}$ mezclada con $\mathrm{H}_{2} \mathrm{SO}_{4}$ ). El extracto se cromatografió en una columna de sílica ácida/básica/con nitrato de plata $\left(\mathrm{AgNO}_{3}\right)$ y en una segunda columna de sílica con carbón activado. Cuando existió interferencia con éteres difenílicos clorados, se cromatografió en una tercera columna de alúmina básica. El extracto final se enriqueció con una solución que contenía cantidades especificadas de dos estándares de recuperación de PCDD marcados isotópicamente (Wellington Laboratories Inc., Guelph, Ontario, Canadá). Se obtuvo un volumen final de $20 \mu \mathrm{L}$. El análisis HRGC/HRMS fue realizado con un cromatógrafo de gases Hewlett Packard ${ }^{\circledR}$ HP 5890 serie II, acoplado con espectrómetro de masas de alta resolución VG Autospec ${ }^{\circledR}$, resolución $>10.000$, con monitoreo de iones específicos multigrupo; volumen de inyección $1 \mu \mathrm{L}$; columna de cromatografía de gases Rtx-Dioxin $260 \mathrm{~m}$ x 0,25 mm x 0,25 $\mu \mathrm{m}$ (Restek). La cuantificación fue realizada usando estándares internos y los resultados fueron corregidos por recuperaciones de estándares internos.

\section{ANÁLISIS ESTADÍSTICO}

Se utilizaron los programas estadísticos Minitab ${ }^{\circledR}$ versión 15.0 para Windows (Minitab Inc, State College, PA, USA), S-Plus ${ }^{\circledR}$ versión 6.4 para Windows (TIBCO software Inc, Somerville, MA, USA) y SigmaPlot ${ }^{\circledR}$ versión 11.0 para Windows (Systat Software Inc, San Jose, CA, USA). Una vez obtenidos los resultados expresados en pg WHO-TEQ/g de grasa para HRGC/HRMS y pg TCDD$\mathrm{EQs} / \mathrm{g}$ de tejido para EROD-H4IIE, fueron representados en un gráfico de dispersión (figura 1). Se propuso un modelo para explicar la relación entre los resultados del bioensayo EROD-H4IIE y del análisis cromatográfico. Para su ajuste se aplicó el modelo: $Y_{i}=\beta_{0}+\beta_{1} x_{i}+\beta_{2} x_{i}^{2}+\cdots+\beta_{\mathrm{p}} x_{i}^{\mathrm{p}}+$ $\varepsilon_{i}$, donde, $Y_{i}$ son los valores obtenidos de HRGC/HRMS, $x_{i}$ los obtenidos por EROD-H4IIE, $\beta_{i}$ los coeficientes del modelo de regresión y $\varepsilon_{i}$ corresponde al error aleatorio.

\section{RESULTADOS}

En el cuadro 1 se observa que el plantel 4 (P4) tiene un promedio de los resultados obtenidos por HRGC/HRMS mayor al resto, con una desviación estándar (DE) elevada, coherente con lo obtenido en el bioensayo EROD-H4IIE. Se realizó un Anova entre planteles, cuyo resultado nos indica que existen diferencias entre planteles y los intervalos de confianza simultáneos de Tukey al 95\% muestran que esta diferencia está dada por P4. 
Cuadro 1. Resultados promedio obtenidos por el bioensayo EROD-H4IIE y por HRGC/HRMS. and by HRGC/HRMS

Average results obtained by the EROD-H4IIE bioassay

\begin{tabular}{lccccc}
\hline & $\begin{array}{c}\text { HRGC/HRMS } \\
\text { (WHO-TEQ/g de } \\
\text { grasa) }\end{array}$ & \multicolumn{3}{c}{$\begin{array}{c}\text { H4IIE } \\
\text { (pg TCDD-EQ/g de } \\
\text { tejido) }\end{array}$} \\
\hline Plantel & Promedio & DE & Promedio & DE & $\mathrm{n}$ \\
\hline P1 & 0,58 & 0,17 & 0,64 & 0,81 & 6 \\
P2 & 0,57 & 0,21 & 1,39 & 1,34 & 13 \\
P3 & 0,40 & 0,19 & 0,75 & 0,73 & 15 \\
P4 & 2,61 & 1,99 & 4,67 & 3,67 & 7 \\
\hline
\end{tabular}

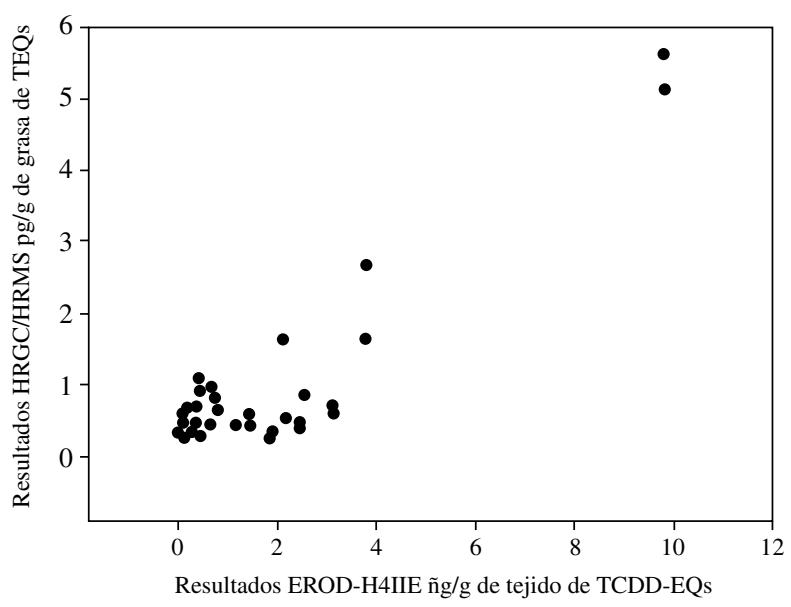

Figura 1. Gráfico de dispersión de resultados EROD-H4IIE expresado en pg/g de tejido de TCDD-EQs contra el resultado de HRGC/HRMS expresado en pg/g de grasa de TEQs, para 41 muestras de trutro de pollo. Se observa una buena correlación $(r=0,878)$, sin embargo, en la mayoría de los datos existe una sobrestimación de los resultados obtenidos por bioensayo ERODH4IIE por sobre los obtenidos por HRGC/HRMS.

Scatter plot for EROD-H4IIE results expressed in $\mathrm{pg} / \mathrm{g}$ tissue of TCDD-EQs vs HRGC/HRMS the results expressed in pg/g fat of TEQs, for 41 sample of broiler chicken drumstick. The data reveal a good correlation $(r=0.878)$, however, in most of the data there is an overestimation of the results obtained by EROD-H4IIE bioassay above the ones obtained by HRGC/HRMS.

\section{ANÁLISIS DE MODELOS}

Se consideraron 2 modelos bajo el principio de parsimonia: modelo 1 (M1): $Y_{i}=\beta_{0}+\beta_{1} x_{i}+\varepsilon_{i}$ y modelo 2 (M2): $Y_{i}=\beta_{0}+\beta_{1} x_{i}+\beta_{2} x_{i}^{2}+\varepsilon_{i}$. Los resultados del análisis de varianza de M1, muestran que bajo la prueba $\mathrm{F}$ de Fisher el modelo es significativo $(\mathrm{P}<0,0001)$, con un coeficiente de determinación $\left(\mathrm{R}^{2}\right)$ de 0,770 . Bajo la prueba $t$ de Student para la significancia de los coeficientes de M1, se obtuvo un valor P no significativo para el intercepto $(\mathrm{P}>0,05)$, por lo que este coeficiente fue retirado del modelo. El resultado de análisis de varianza de M2 muestra que la prueba F de Fisher es significativa $(\mathrm{P}<0,0001)$. Para este modelo el coeficiente de determinación ajustado ( $\mathrm{R}^{2}$-ajustado) es de 0,879 . $\mathrm{Al}$ analizar los resultados de las estimaciones para los parámetros $\mathrm{M} 2$, bajo la dócima de $t$ de Student, muestra que el coeficiente asociado a EROD-H4IIE no es significativo $(\mathrm{P}>0,95)$ en el modelo, por lo cual fue retirado. Es importante destacar que bajo la misma dócima t de Student, el coeficiente de intercepto como el coeficiente asociado a EROD-H4IIE al cuadrado son significativos $(\mathrm{P}<0,001)$ para el modelo propuesto. Como consecuencia de lo anterior se propusieron 2 nuevos modelos: modelo 3 (M3): $Y_{i}=\beta_{1} x_{i}+\varepsilon_{i}$ y modelo 4 (M4): $Y_{i}=\beta_{0}+\beta_{2} x_{i}^{2}+\varepsilon_{i}$. Del análisis de M3 se puede observar que para la prueba $F$ de Fisher se obtuvo un valor $F$ significativo $(P<0,0001)$, con un $R^{2}$ de 0,849 . Bajo la prueba $t$ de Student para la nulidad del coeficiente, se puede destacar que éste es significativo en M3 $(\mathrm{P}<0,0001)$. Se realizó el mismo análisis para el modelo 4 obteniendo los mismos resultados tanto para el análisis de varianza como para la estimación de los parámetros, con un $\mathrm{R}^{2}$ de 0,885 .

Con el objetivo de seleccionar el mejor modelo que permitiera realizar una equivalencia entre ambas técnicas, se consideraron los siguientes criterios: Criterio de Información de Akaike (AIC) (Akaike 1973, Anderson y col 1994) y el Criterio de Información Bayesiano (BIC) (Posada y Rosero 2007). Del análisis precedente se concluyó que el modelo más adecuado es M4. De esta forma el modelo estimado es:

$$
\text { HRGC/HRMS }=0,481+0,051[\text { EROD-H4IIE }]^{2}
$$

El gráfico de ajuste de M4 (figura 2) muestra las estimaciones de los niveles determinados por HRGC/ HRMS contra EROD-H4IIE y el límite superior para la estimación a un $95 \%$ y $99 \%$ de confianza, en carne de pollo, donde se puede determinar que, si se obtiene un valor en EROD-H4IIE de 2,2 pgTCDD-EQ/g de tejido se estima que corresponderá a $0,73 \mathrm{pg}$ WHO-TEQ/g de grasa en HRGC/HRMS. Además, para el mismo valor de EROD-H4IIE con un 99\% de confianza, se obtendrá una estimación como límite superior que equivaldrá a 0,83 pg WHO-TEQ/g de grasa en HRGC/HRMS.

\section{ANÁLISIS DE SENSIBILIDAD}

Se realizó un análisis de sensibilidad para las estimaciones de los coeficientes del modelo de regresión estimado, tanto para el intercepto $\left(\beta_{0}\right)$ como para el coeficiente asociado a EROD-H4IIE al cuadrado $\left(\beta_{2}\right)$. En el histograma de las estimaciones de $\beta_{0}$ (figura 3 ) se muestra la estabilidad de este parámetro durante el proceso de análisis. En la figura se observa que el comportamiento es asintóticamente normal, compacto, centrado y con un ligero sesgo hacia la izquierda, pero con una estimación bastante estable para el 


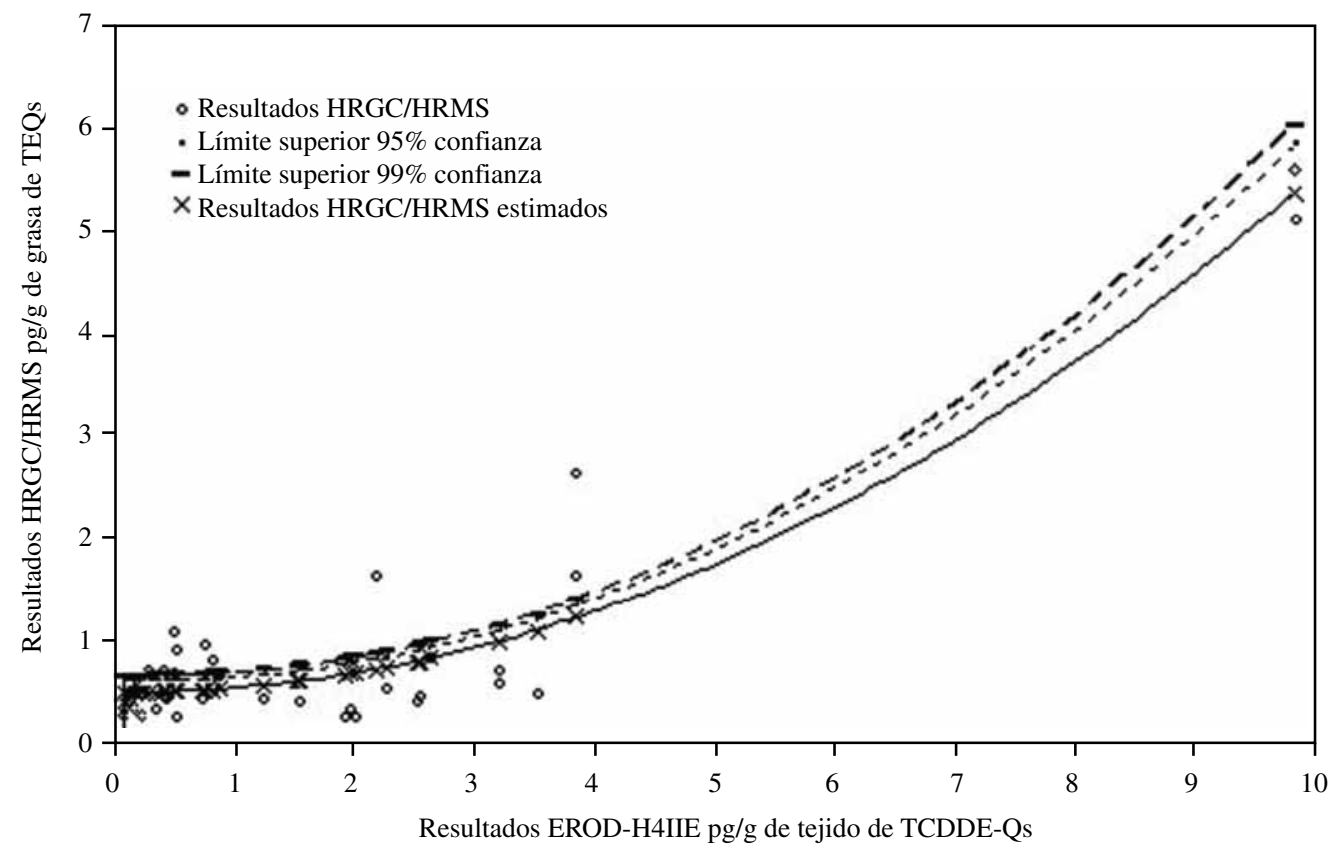

Figura 2. Gráfico de ajuste de los resultados estimados por M4, donde se muestra la equivalencia de los resultados entre el bioensayo EROD-H4IIE y HRGC/HRMS y las estimaciones para los límites superiores con $95 \%$ y 99\% de confianza.

Adjustment plot of the estimates results for M4, were the equivalence of the results between the EROD-H4IIE Bioassay and HRGC/HRMS and the estimations for the upper limits with $95 \%$ and $99 \%$ confidence is shown.

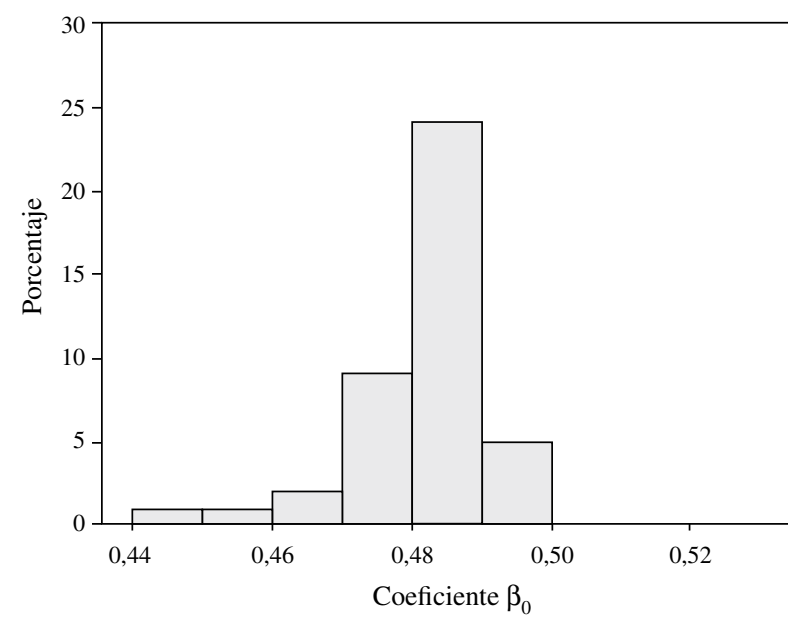

Figura 3. Histograma para la distribución de las estimaciones del coeficiente $\beta_{0}$ en M4, expresadas como porcentaje, donde se puede observar el comportamiento para las estimaciones y estabilidad para el modelo y conjunto de datos.

Histogram for the distribution of the $\beta_{0}$ coefficient estimation in M4, expressed as percentage, where the behavior and stability for the model and data set is shown.

coeficiente y el conjunto de datos. En el histograma de las estimaciones asociadas a $\beta_{2}$ (figura 4 ) se puede observar una distribución asintóticamente normal, mucho más compacta y centrada que para $\beta_{0}$ y con valores extremos a ambos lados de la distribución, lo que sugiere una distribución con colas más pesadas ( $\mathrm{t}$ de Student).

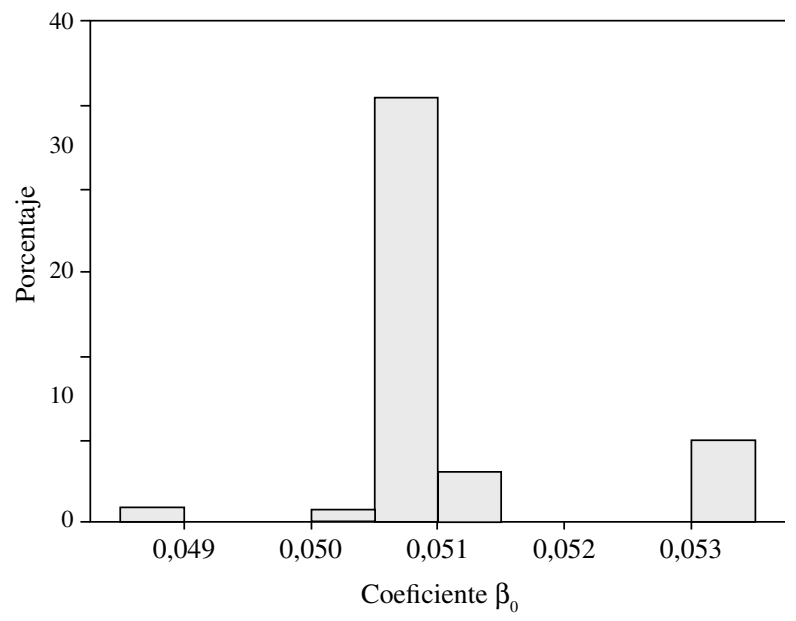

Figura 4. Histograma para la distribución de las estimaciones del coeficiente $\beta_{2}$ en $M 4$, expresadas como porcentaje, donde se puede observar el comportamiento para las estimaciones y estabilidad para el modelo y conjunto de datos.

Histogram for the distribution of the $\beta_{2}$ coefficient estimation in M4, expressed as percentage, where the behavior and stability for the model and data set is shown.

\section{DISCUSIÓN}

La utilización del bioensayo con línea celular H4IIE ofrece una herramienta útil, alternativa y/o complementaria para estimar la presencia de PCDDs, PCDFs y DL-PCBs en carnes de pollo además de otras matrices. Según Whyte 
y col (2004) entre las ventajas que posee el bioensayo se encuentran: 1) Utiliza un mecanismo de acción común para la detección de dioxinas, la presencia de compuestos inductores del citocromo P4501A1 en el extracto es medida en conjunto, además el bioensayo puede evaluar estos compuestos aun en concentraciones menores al límite de detección del análisis químico; 2) al analizar de manera simultánea los compuestos inductores del citocromo P4501A1, el bioensayo integra interacciones entre los diferentes congéneres presentes en el extracto; 3 ) el bioensayo es más económico que la HRGC/HRMS (10-25\%).

Como ejemplo de uso del bioensayo, el US Geological Survey (USGS) ha ejecutado el programa Biomonitoring of Environmental Status and Trends (BEST) en las cuencas del río Yukon, Columbia, Río Grande y Mississippi, con el fin de detectar contaminantes orgánicos persistentes (COPs) y disruptores endocrinos. Dicho programa se ha llevado a cabo en parte a través de la aplicación del Bioensayo EROD-H4IIE en peces (USGS 2002, USGS $2004^{\mathrm{a}}$, USGS $2004^{\mathrm{b}}$, USGS $2004^{\mathrm{c}}$ ). Este programa demuestra que es factible utilizar dicho bioensayo a gran escala, con óptimos resultados y ha permitido identificar las áreas contaminadas de las cuencas, así como aquellas con problemas emergentes, permitiendo además evaluar el éxito de las actividades de remediación (USGS 2004c). Las ventajas del bioensayo han permitido que sea utilizado para evaluar la potencia de los extractos que contienen hidrocarburos halogenados planares $(\mathrm{PHH})$, provenientes de 22 especies de peces, 14 especies de aves y 4 especies de mamíferos (Whyte y col 2004).

En el presente trabajo se puede observar que los resultados promedio obtenidos por el bioensayo EROD-H4IIE (cuadro 1) tienden a ser mayores a los de HRGC/HRMS, comportamiento similar al obtenido por Till y col (1997) en un estudio efectuado en cenizas. Esta diferencia entre ambas técnicas se puede deber a que en el bioensayo los hidrocarburos aromáticos policíclicos (PAHs) y componentes no identificados posean actividad similar a dioxinas (Chaloupka y col 1993) y a la presencia de DL-PCBs, los cuales no fueron analizados mediante HRGC/HRMS. Respecto a esto último, el aporte a la toxicidad final o a su valor de equivalente tóxicos (TEQs) en el extracto es muy reducido, considerando sus bajos valores de factores de equivalencia tóxica (TEFs) (van den Berg y col 1998).

A pesar de que existen otros bioensayos muy eficientes como DR CALUX ${ }^{\circledR}$, XDS CALUX $^{\circledR}$ o DR-EcoScreen ${ }^{\circledR}$ (Behnisch y col 2002, Hoogenboom 2002, Denison y col 2004, Anezaki y col 2009), para los dos primeros se necesita adquirir la licencia a un costo oneroso, la cual debe renovarse anualmente, adquirir los equipos, capacitación y un pago por número de muestras analizadas a las empresas que comercializan dichos bioensayos (BioDetectionSystems) ${ }^{3}$. El bioensayo con células DR-EcoScreen ${ }^{\circledR}$ fue desarrollado por

\footnotetext{
http://wwww.biodetectionsystems.com/. Fecha de consulta: 07 de abril de 2010
}

el Instituto de Salud Pública de Hokkaido, Japón (Kojima y col 2010) y tampoco es de libre disposición. En cambio, el bioensayo EROD-H4IIE no requiere del pago de una licencia comercial, por tanto, es una alternativa adecuada para realizar estudios de biomonitoreos a gran escala.

Con respecto al modelo de equivalencia, éste se ajusta a la tendencia del gráfico de dispersión de la figura 1. Además éste tiene un nivel inicial de 0,481 (cuando el resultado de H4IIE es cero), es decir, considera un nivel basal de concentración de PCDDs, PCDFs y DL-PCBs. Para los valores de las estimaciones asociadas a $\beta_{2}$ el efecto sobre los resultados es de un 5,1\%. En general, es un modelo significativo para todos sus parámetros y coeficientes y que bajo los criterios de selección es el más adecuado entre los otros modelos propuestos.

Al comparar el presente estudio con el realizado por Wan y col (2004) se puede observar que en ambos estudios hay un modelo de equivalencia propuesto para EROD-H4IIE contra HRGC/HRMS, que para el caso de Wan y col es un modelo lineal. Además ambos modelos proponen un nivel basal de concentración de dioxinas, furanos y DL-PCBs. En el modelo propuesto por Wan y col el nivel basal es menor que en el presente estudio y $\mathrm{R}^{2}$ es mayor. Esta diferencia podría estar dada por el pequeño tamaño de muestra (6 muestras) señalado en dicho trabajo y también por el tipo de matriz analizada (sedimentos, colina clorada, desperdicio electrolítico, benceno quinona tetraclorada, cenizas y suelo). Este tamaño de muestra también podría explicar la diferencia en el tipo de modelo, que para Wan y col es lineal y el del presente estudio es cuadrático.

Till y col (1997) encontraron en extractos de cenizas de incineradores de basura, madera, crematorios y de reciclado de metales, valores de correlación semejantes al presente estudio. La correlación en cenizas fue de 0,750 y 0,900 dependiendo del origen de la matriz, mientras que en el actual estudio para los extractos de las muestras de pollos broiler fue de 0,878 (figura 1).

El bioensayo con línea celular H4IIE entrega estimaciones razonables y confirma que la inducción de CYP1A está dada principalmente por PCDD/Fs y, por tanto, es una técnica útil para el biomonitoreo de estos contaminantes y puede ser aplicado como método de cribado en sistemas de producción animal y específicamente en producción de pollos broiler, aunque todo resultado positivo debe ser analizado mediante HRGC/HRMS como método confirmatorio de identidad.

\section{RESUMEN}

En el presente estudio se aplicó el bioensayo EROD con línea celular H4IIE para determinar la equivalencia de resultados para carnes de pollo, entre el bioensayo EROD-H4IIE en pg TCDD-EQ/g de tejido y los resultados de la cromatografía de gases de alta resolución acoplada a espectrometría de alta resolución (HRGC/HRMS) en pg WHO-TEQ/g de grasa. Para lo anterior se utilizaron 41 muestras compuestas de trutros de pollo. Éstas fueron obtenidas en la planta faenadora de 4 planteles diferentes de Chile, durante el beneficio de animales (38 y 43 días de 
edad) entre los años 2004 y 2007. Cada muestra fue analizada mediante ambas técnicas analíticas. Con los resultados se determinó un modelo de regresión para la equivalencia de ambas técnicas. De esta forma el modelo obtenido fue: HRGC/HRMS $=0,481+0,051$ [EROD-H4IIE] ${ }^{2}$, $\mathrm{R}^{2}=0,885$, en que para un valor en EROD-H4IIE de 2,2 pg TCDD-EQ/g de tejido se estima que corresponderá a $0,73 \mathrm{pg} \mathrm{WHO}-\mathrm{TEQ} / \mathrm{g}$ de grasa en HRGC/HRMS. Además, para el mismo valor de EROD-H4IIE con un 95\% de confianza, se obtendrá una estimación como límite superior que equivaldrá a 0,83 pg WHO-TEQ/g de grasa en HRGC/HRMS. Al tomar el mismo valor de EROD-H4IIE con un $99 \%$ de confianza, se estima un límite superior que equivaldrá a $0,88 \mathrm{pg}$ WHO-TEQ/g de grasa en HRGC/HRMS. De esta manera se concluye que el bioensayo EROD-H4IIE puede ser aplicado como método de cribado en sistemas de producción animal y específicamente en producción de pollos broiler.

\section{AGRADECIMIENTOS}

A Columbia Enviromental Research Center (CERC) del US Geological Survey, en especial al Dr. Donald Tillitt, a Diane Nicks y Mike Tanner, a la Asociación de Productores Avícolas de Chile A.G, a la Comisión Nacional de Investigación Científica y Tecnológica (CONICYT) y a Hugo Schöffer.

\section{REFERENCIAS}

Akaike H. 1973. Information theory and an extension of the maximum likelihood principle. In: Petrov BN, Csa'ki F (eds). Second International Symposium on Information Theory. Akadémiai Kiadi, Budapest, Hungary, Pp 267-281.

Anderson DR, KP Burnham, CG White. 1994. AIC model selection in overdispersed capture-recapture data. Ecology 75, 1780-1793.

Anezaki K, K Yamaguchi, S Takeuchi, M Iida, K Jin, H Kojima. 2009. Application of a bioassay using DR-EcoScreen cells to the determination of dioxins in ambient air: a comparative study with HRGC-HRMS analysis. Environ Sci Technol 43, 7478-7483.

Behnisch PA, K Hosoe, A Brouwer, S Sakai. 2002. Screening of dioxinlike toxicity equivalents for various matrices with wild type and recombinant rat hepatoma H4IIE cells. Toxicol Sci 69, 125-130.

Chaloupka K, N Harper, V Krishnan, M Santostefano, LV Rodriguez, S Safe. 1993. Synergistic activity of polynuclear aromatic hydrocarbon mixtures as aryl hydrocarbon (Ah) receptor agonists. Chem Biol Interact 89, 141-158.

Covaci A, S Voorspoels, P Schepens, P Jorens, R Blust, H Neels. 2008. The Belgian PCB/dioxin crisis- 8 years later. An overview. Environ Toxicol and Pharmacol 25, 164-170.

Denison MS, B Zhao, DS Baston, GC Clark, H Murata, H Dalho. 2004. Recombinant cell bioassay for the detection and relative quantification of halogenated dioxins and related chemicals. Talanta 63 , 1123-1133.

Egeland GM, MH Sweeney, MA Fingerhut, KK Wille, TM Schnorr, WE Halperi. 1994. Total serum testosterone and gonadotropins in workers exposed to dioxin. Am J Epidemiol 139, 272-281.

European Commission. 2001. Opinion of the Scientific Committee on food on the risk assessment of dioxins and dioxin-Like PCBs in food. Update based on new scientific information available since the adoption of the SCF opinion of $22^{\text {nd }}$ November 2000. Adopted on 30 May 2001. Health \& Consumer Protection Directorate-General. Directorate C - Scientific Opinions.C2 - Management of scientific committees II; scientific co-operation and networks.

Fingerhut MA, WE Halperin, DA Marlow, LA Piacitelli, PA Honchar, MH Sweeney, AL Greife, PA Dill, K Steenland, AJ Surusa. 1991. Cancer mortality in workers exposed to 2,3,7,8 tetrachlorodibenzop-dioxin. N Engl J Med 324, 212-218.

Guo YL, ML Yu, CC Hsu. 2003. The Yucheng Rice Oil Poisoning Incident. In: Schecter A, Gasiewicz TA (eds). Dioxins and Health. John Wiley and Sons, Inc., Hoboken, New Jersey, USA, Pp 893-920.
Hayward D, D Norttrup, A Gardner, M Clower Jr. 1999. Elevated TCDD in chicken eggs and farm-raised catfish fed a diet with ball clay from a southern United States mine. Environ Res A 81, 248-56.

Hoogenboom R. 2002. The combined use of CALUX bioassay and the HRGC-HRMS method for the detection of novel dioxin sources and new dioxin-like compounds. Environ Sci Pollut R 9, 304-306.

Kennedy SW, SP Jones. 1994. Simultaneous measurement of cytochrome P4501A catalytic activity and total protein concentration with a fluorescence plate reader. Anal Biochem 222, 217-223.

Kojima H, S Takeuchi, T Nagai. 2010. Endocrine disrupting potential of pesticides via nuclear receptors and aryl hydrocarbon receptor. J Health Sci 56, 374-386.

Longnecker MP, JE Michalek. 2000. Serum dioxin level in relation to diabetes mellitus among Air Force veterans with background levels of exposure. Epidemiology 11, 44-48.

Mason G, T Sawyer, B Keys, S Bandiera, M Romkes, J PiskorskaPliszczynska, B Zmudzka, S Safe. 1985. Polychlorinated dibenzofurans (PCDFs): correlation between in vivo and in vitro structure-activity relationships. Toxicology 37, 1-12.

Nakanishi Y, N Shigematsu, Y Kurita. 1985. Respiratory involvement and immune status in Yusho patients. Environ Health Persp 59, 31-36.

Nicks DK, DE Tillitt. 2003. H4IIE bioassay-derived 2,3,7,8-tetrachlorodibenzo-p-dioxin equivalents (TCDD-EQ) in fish collected in 2000 from large estuaries along the western coast of the United States. USGS, Columbia Environmental Research Center, Biochemistry \& Physiology Branch, Final laboratory report FY 2003-30-01, 2003.

Okey A, M Franc, I Moffat, N Tijet, P Boutros, M Korkalainen, J Tuomisto, R Pohjanvirta. 2005. Toxicological implications of polymorphisms in receptors for xenobiotic chemicals: the case of the aryl hydrocarbon receptor. Toxicol Appl Pharm 207, 43-51.

Posada SL, R Rosero. 2007. Comparación de modelos matemáticos: una aplicación en la evaluación de alimentos para animales. Rev Col Cienc Pec 20, 141-148.

Richter CA, DE Tillitt, M Hannink. 2001. Regulation of subcellular localization of the aryl hydrocarbon receptor (AhR). Arch Biochem Biophys 389, 207-217.

Roeder RA, MJ Garber, GT Schelling. 1998. Assessment of Dioxins in Foods from Animal Origins. J Anim Sci 76, 142-151.

Saegerman C, L Pussemier, A Huyghebaert, ML Scippo, D Berkvens. 2006. On-farm contamination of animals with chemicals contaminants. Rev Sci Tech Off Int Epiz 25, 655-673.

Spiro TG, M Stigliani. 2004. Sustancias químicas tóxicas. En: Spiro TG, Stigliani M (eds). Química Ambiental. 2a ed. Pearson Educación SA, Madrid, España, Pp 413-460.

Sweeney MH, P Mocarelli. 2000. Human health effects after exposure to 2,3,7,8-TCDD. Food Additives and Contaminants 17, 303-316.

Till M, P Behnisch, H Hagenmaier, KW Bock, D Schrenk. 1997. Dioxinlike components in incinerator fly ash: a comparison between chemical analysis data and results from a cell culture bioassay. Environ Health Persp 105, 1326-1332.

Tillitt DE, JP Giesy, GT Ankley. 1991. Characterization of the H4IIE rat hepatoma cell bioassay as a tool for assessing toxic potency of planar halogenated hydrocarbons in environmental samples. Environ Sci Technol 25, 87-92.

Tillitt DE, TJ Kubiak, GT Ankley, JP Giesy. 1993. Dioxin-like toxic potency in Forster's Tern eggs from Green Bay, Lake Michigan, North America. Chemosphere 26, 2079-2084.

Unión Europea. 2006. Reglamento (CE) N $1883 / 2006$ de la Comisión de 19 de enero de 2006 por el que se establecen métodos de muestreo y análisis para el control oficial de los niveles de dioxinas y PCB similares a las dioxinas en determinados productos alimenticios. Diario Oficial de la Unión Europea L 364/32. ES. 20.12.2006.

USGS, United States Geological Survey. 2002. Biomonitoring of Environmental Status and Trends (BEST) Program: Environmental Contaminants and their Effects on Fish in the Mississippi River Basin. Biological Science Report USGS/BRD/BSR-2002-0004.

USGS, United States Geological Survey. 2004 . Biomonitoring of environmental status and trends (BEST) program: environmental 
contaminants and their effects on fish in the Yukon River basin. Scientific Investigations Report 2004-5285.

USGS, United States Geological Survey. 2004 ${ }^{\mathrm{b}}$. Biomonitoring of environmental status and trends (BEST) program: environmental contaminants and their effects on fish in the Columbia River basin. Scientific Investigations Report 2004-5154.

USGS, United States Geological Survey. 2004c . Biomonitoring of Environmental Status and Trends (BEST) Program: Environmental contaminants and their effects on fish in the Rio Grande basin. Scientific Investigations Report 2004-5108.

Valdovinos CE. 2009. Dioxinas y furanos en Chile: Riesgos potenciales de contaminación en la producción avícola y porcina. Monografía, Programa de Doctorado en Ciencias Silvoagropecuarias y Veterinarias. Campus Sur, Universidad de Chile, Santiago, Chile, Pp 3-4.

van den Berg M, L Birnbaum, BTC Bosveld, B Brunström, B Cook, M Feeley, JP Giesy, A Hanberg, R Hasegawa, SW Kennedy,
T Kubiak, JC Larsen, FS van Leeuwen, AK Dijien Liem, C Nolt, RE Peterson, L Poellinger, S Safe, D Schrenk, D Tillitt, M Tysklind, M Younes, F Waern, T Zacharewski. 1998. Toxic Equivalency Factors (TEFs) for PCBs, PCDDs, PCDFs for Humans and Wildlife. Environ Health Persp 106, 775-792.

Wan X, T Ma, W Wu, Z Wang. 2004. EROD activities in a primary cell culture of grass carp (Ctenopharyngodon idellus) hepatocytes exposed to polychlorinated aromatic hydrocarbons. Ecotox Environ Safe 58, 84-89.

Weisglas-Kuperus N, S Patandin, G A Berbers, TC Sas, P G Mulder, P J Sauer, H Hooijkaas. 2000. Immunologic effects of background exposure to polychlorinated biphenyls and dioxins in Dutch preschool children. Environ Health Persp 108, 1203-1207.

Whyte JJ, CJ Schmitt, DE Tillitt. 2004. The H4IIE Cell bioassay as an indicator of dioxin-like chemicals in wildlife and the environment. Crit Rev Toxicol 34, 1-83. 